\title{
Design of a Novel Composite Dielectric Metallic Structure Reflectarray
}

\author{
Tingzhou Jiang ${ }^{1, ~ a, ~ H u ~ Y a n g ~}{ }^{1}$,Shaojun $\mathrm{Mao}^{2}$, Lei Gu, Fei Zhao ${ }^{2}$ \\ ${ }^{1}$ College of School of Electronic Science and Engineering, National University of Defense \\ Technology, Changsha, 410073, Hunan province, China \\ ${ }^{2}$ Southwest Electronics and Telecommunication Technology Research Institute, Chengdu, 610041, \\ Sichuan province, China \\ ajiangtz09@163.com
}

Keywords: reflectarray, phase shift, bandwidth

Abstract. In this paper, a novel hybrid structure of reflectarray with dielectric cylinder, metallic cylinder and metallic ground is designed. This structure can generate a pencil beam in a desired direction by adjusting its phase appropriately with its $1-\mathrm{dB}$ gain bandwidth achieving $22.56 \%$. Moreover, radiation patterns of the structure at different frequencies are in good consistency.

\section{Introduction}

In recent years, large-aperture antennas have been widely used in high-gain applications such as radars, satellite communications, radio astronomy and deep-space communications. Traditionally, high-gain antennas have relied on conventional parabolic reflector antennas and phased array antennas. However, in many cases, both of them have some drawbacks. Due to these disadvantages, a new kind antenna named reflectarray was introduced by D.G. Berry in 1963 [1]. Reflectarray combines advantages of conventional parabolic reflector antennas and phased array antennas [2].Compared to phased array antennas, reflectarray without complicated feeding network which will produce loss has superior radiant efficiency [3].Compared to conventional parabolic reflector antennas, major advantages of reflectarray without curved surface are small volume ,low profile, light weight and easy folding [4].

Due to the properties of lower loss, wider bandwidth and higher radiation efficiency of dielectric resonator antennas [5], dielectric reflectarray has been introduced by some scholars. However the bandwidth of the dielectric reflectarray is relatively narrow. Papers [6,7] show that the 1-dB bandwidth of the dielectric reflectarrays is only $10 \%, 13 \%$, and the $1.5-\mathrm{dB}$ bandwidth of the dielectric reflectarray is only $15 \%$ in paper [8]. In order to improve the bandwidth of dielectric reflectarray, a hybrid structure reflectarray with dielectric and metal is designed in this paper. The bandwidth of the reflectarray with 208 elements is $22.56 \%$ which is wider than above.

\section{Design of the reflectarray elements}

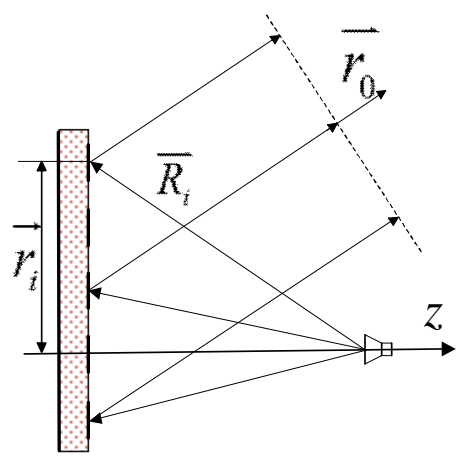

Fig. 1 Structure of reflectarray

A reflectarray consists of a quasi-periodic structure of illuminating feed and resonant elements, the structure of the reflectarray is represented in Fig. 1. When illuminated by feed, the element with a 
certain phase-shift is designed to produce a desired beam. Similar to conventional parabolic reflector antennas, the phase shift of the field reflected from each element must be the same in a desired direction. By appropriately adjusting the phase, a pencil beam can be generated in a desired direction.

Designing the size of each element to compensate the phase delay from the feed to the aperture plane to establish equiphase surface is of vital importance. The constant phase delay $\varphi_{i}$ can be given by Eq. 1:

$$
\varphi_{i}=2 n \pi+k_{0}\left(R_{i}-r_{i} r_{0}\right), n=0,1,2 \mathrm{~L}
$$

Where $k_{0}$ is free space wavenumber, $R_{i}$ is the distance from the phase center of feed to the center of the ith element and $r_{0}$ is the position vector of the center of ith element from array center.

At the same time, the phase shift generated by each element must be restricted to $0 \sim 360^{\circ}$. There are three methods for elements to achieve the desired phase shift. Firstly, using elements with different phase delay lines [9]; secondly, adjusting element rotation [10]; thirdly, altering elements with variable size [11].In this paper, the third method is adopted and the element is shown in Fig. 2(a).The element consists of dielectric cylinder, metallic cylinder and metallic ground. Fig. 2(b) and Fig. 2(c) show the top view and side view of the element respectively.

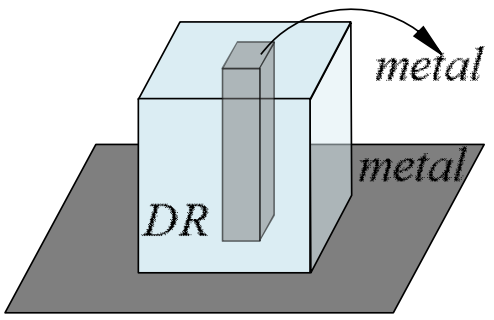

(a)

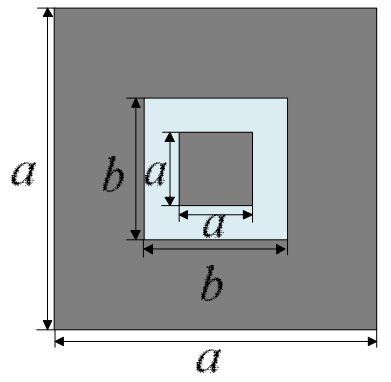

(b)

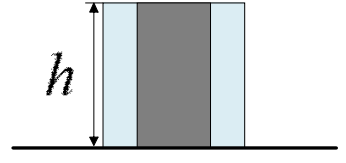

(c)

Fig. 2 (a ) Structure of the element (b) top view of the element (c) side view of the element

The space $a$ between different elements and the angle $\theta$ of incident wave should meet the following Eq. 2 [12]:

$$
a<\frac{\lambda}{1+\sin \theta}
$$

Where $\lambda$ represents free space wavelength.

In this paper, $f=13 \mathrm{GHz}, \theta=32^{\circ}$, then $\lambda \approx 23.08 \mathrm{~mm}$. According to Eq. $2, a<15.38 \mathrm{~mm}$. Thus, set $a=10 \mathrm{~mm}(0.43 \lambda)$.

Appropriately designing and optimizing the dimension of the element in CST, we can get the dielectric constant $\varepsilon=10.2$, the width of dielectric is $b=6$, the width of metal is $c=k \times b, k=0.4$. Then, we can obtain a smooth phase curve with the range over $360^{\circ}$ at different frequencies $(10 \mathrm{GHz}-14 \mathrm{GHz})$ by changing $h$ from $1 \mathrm{~mm}$ to $15 \mathrm{~mm}$, as shown in Fig. 3 .

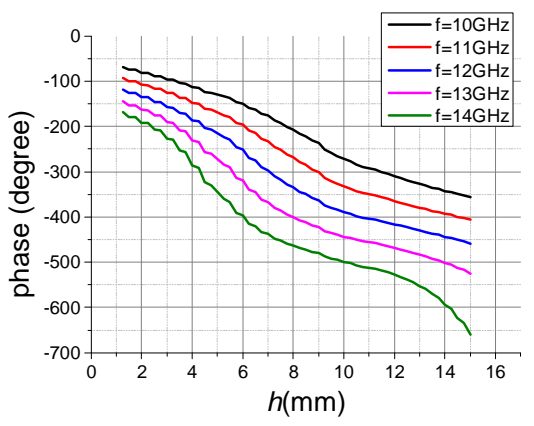

Fig. 3 Phase variation versus size $h$ 


\section{Design of the reflectarray}

Another important part is illuminating feed which influences capability of the reflectarray. Due to high gain and wide bandwidth, horn is widely used as the feed of reflector antennas. Pyramidal horn with aperture size $57.7 \times 36 \times 28 \mathrm{~mm}^{3}$ is designed in this paper. Fig. 4 shows the H-plane and E-plane radiation pattern of the pyramidal horn with its highest gain $14.4 \mathrm{~dB}$ at $13 \mathrm{GHz}$.

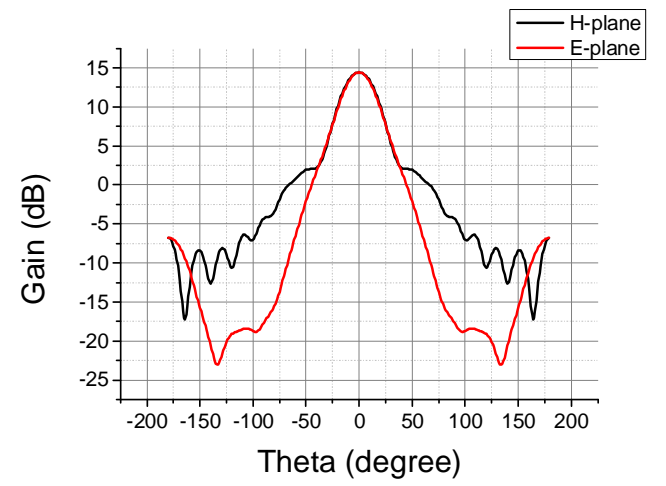

Fig. 4 radiation pattern of the pyramidal horn

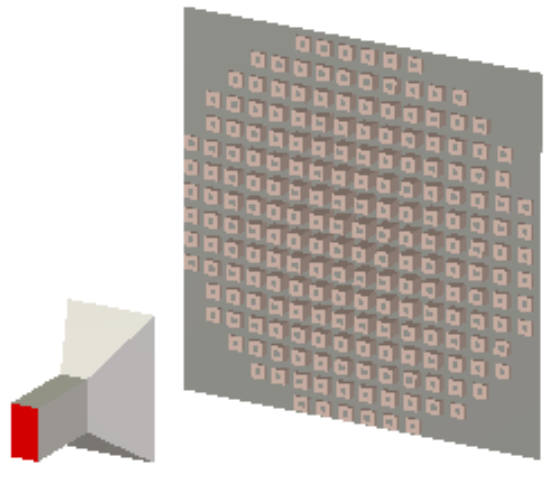

Fig. 5 Simulation model of the reflectarray

Next, devise the height of the radiation element to compensate the phase delay according to the phase curve in Fig. 3. Fig. 5 shows the CST model of the the reflectarray with 208 elements whose aperture is similar to circle. The dimension of the square aperture is $6.88 \lambda \times 6.88 \lambda$. Distance between the phase center of pyramidal horn and the center of the reflectarray antenna is $180 \mathrm{~mm}$ and the focal-length-to-diameter ratio $\mathrm{F} / \mathrm{D}$ is set to 1.125 .

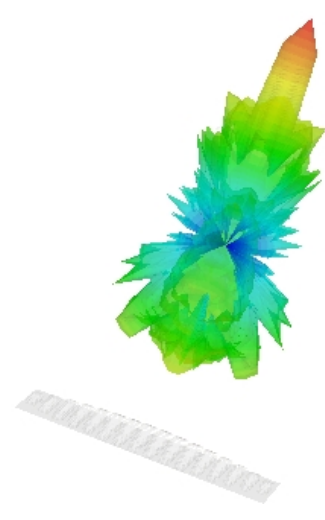

Fig. $63 \mathrm{D}$ radiation pattern at $13 \mathrm{GHz}$

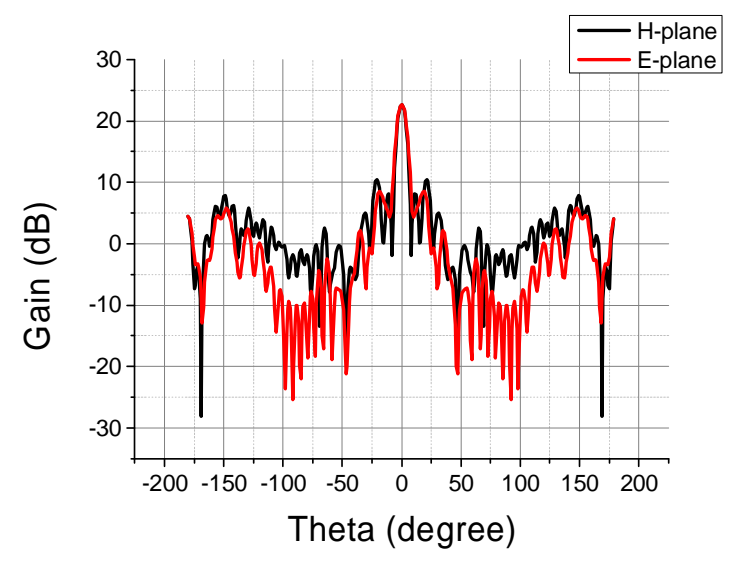

Fig. 7 Radiation pattern at $13 \mathrm{GHz}$

The radiation performance of the reflectarray is obtained using CST and the $3 \mathrm{D}$ radiation pattern at the center frequency $(13 \mathrm{GHz})$ is shown in Fig. 6. It is obvious that the gain of the reflectarray is $22.65 \mathrm{~dB}$ at $13 \mathrm{GHz}$. 


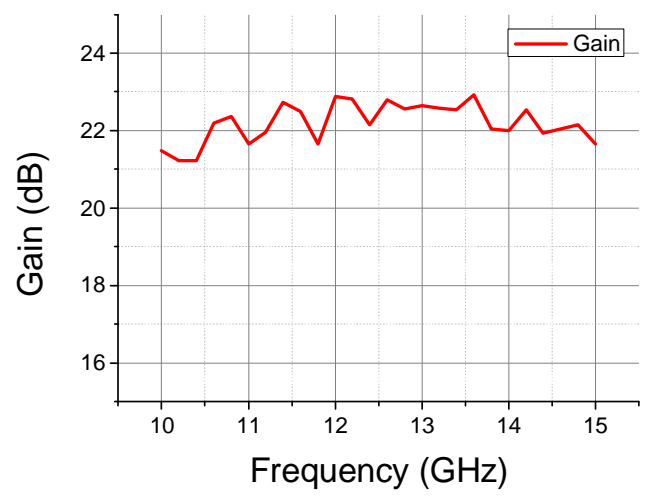

Fig. 8 Gain of the reflectarray versus frequency

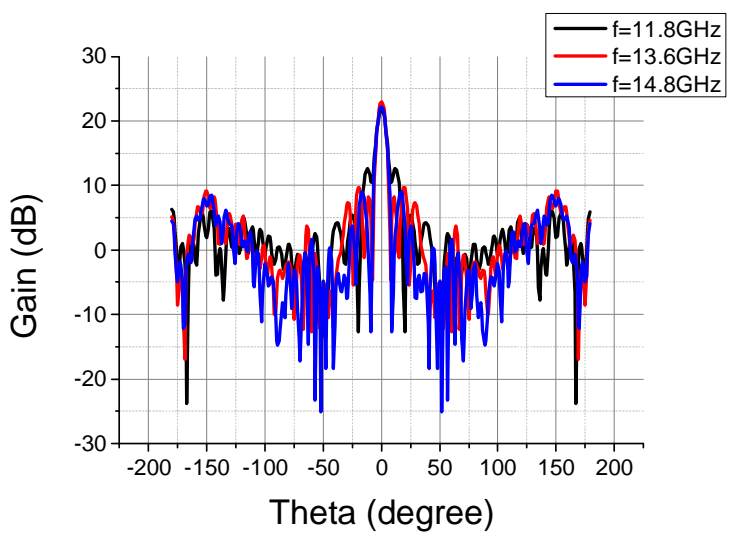

Fig. 9 E-plane radiation pattern

Fig. 7 shows H-plane and E-plane radiation patterns of the reflectarray, evidently the 3-dB beamwidth is $7.6^{\circ}$ and the side-lobe levels is lower than $-14 \mathrm{~dB}$. The gain of the reflectarray versus frequency $(10 \mathrm{GHz}-15 \mathrm{GHz})$ is shown in Fig. 8 and the max gain is $22.92 \mathrm{~dB}$ at $13.6 \mathrm{GHz}$. Obviously $1-\mathrm{dB}$ gain bandwidth of the structure can achieve $22.56 \%(11.8 \mathrm{GHz}-14.8 \mathrm{GHz})$.To demonstrate the radiation performance, a comparison is given in Fig. 9, which shows the E-plane radiation patterns at different frequencies $(11.8 \mathrm{GHz}, 13.6 \mathrm{GHz}$ and $14.8 \mathrm{GHz})$. It is evident that the radiation patterns at different frequencies are in good consistency.

\section{Conclusion}

In this paper a novel composite dielectric metallic structure reflectarray with 208 elements that incorporates a superior phase-shift is design. The gain of the structure is $22.65 \mathrm{~dB}$ at the center frequency and a $22.56 \%$ bandwidth is achieved with $1-\mathrm{dB}$ gain variations. It is shown that the composite dielectric metallic structure reflectarray achieve a wider gain bandwidth than conventional dielectric reflectarray.

\section{References}

[1] Yunxiu Wang, Bingzhong Wang, Hua Li, Huilai Liu, Advances on the research of planar reflectarray antennas, Systems Engineering and Electronics, Vol. 30 No. 2 Feb 2008, In Chinese.

[2] J. Huang, Microstrip reflectarray, IEEE AP-S International Symposium Digital Antennas Propagation, Canda: Ontario, 1991, pp. 612-615.

[3] D.M. Pozar, S.D. Targonski, R. Pokuls, A shaped-beam microstrip patch reflectarray, IEEE Transactions on Antennas and Propagation,Vol.47, July 1999, pp.1167-1173.

[4] Hanqing Ma, Tao Feng, Xin Zhang, Shibo Jiang, Microtrip reflectarray antenna with hybrid elements, Fire control Radar Technology, Vol. 39 No. 3 Sep 2010, In Chinese.

[5] K.M. Luk, K.W. Leung, Dielectric resonator antennas: Overview of the dielectric resonator antenna, Research Studies Press, 2002.

[6] S.H. Zainud-Deen, A.M. Abd-Elhady, A.A. Mitkees, Ahmed A.Kishk, Dielectric Resonator Reflectarray with Two DRA Sizes and Varying Slot Loading, IEEE Antennas and Propagation Society International Symposium (APSURSI), Jul 2010.

[7] M.Abd-Elhady, S.H. Zainud-Deen, A.A. Mitkees, A.A. Kishk,Slot-Loading Rectangular Dielectric Resonator Elements Reflectarray, IEEE Middle East Conference on Antennas and Propagation (MECAP), Oct. 2010. 
[8] A.M. Abd-Elhady, Saber H. Zainude-Deen, A.A. Mitkees, A.A. Kishk, B12. Dual Polarized Dual Feed Aperture-Coupled DRA Reflectarray, 29th National Radio Science Conference (NRSC), Apr 2012 pp. 97-102.

[9] RC Hansen, Microwave Scanning Antennas [M].New York: Academic Press, 1964.

[10] C Han, C Rodenbeck, J Huang and K. Chang. A C/Ka dual frequency dual layer circularly polarized reflectarray antenna with microstripring elements. IEEE Transactions on Antennas and Propagation Antennas Propag, 2004, 52(11): 2871-2876.

[11] S.D. Targonski, D.M. Pozar, Analysis and design of a microstrip reflectarray using patches of variable size. Antennas and Propagation Society International Symposium, Vol. 3 Jun 1994, pp. 1820-1823.

[12] John Huang, Jos Antronio Encinar, Reflectarray Antennas [M]. John Wiley, 2007. 